\title{
Efecto de la arteterapia en la ansiedad y depresión, la capacitación sociocultural y la reducción de la reincidencia penitenciaria de personas reclusas
}

\author{
Pilar Ma DOMÍNGUEZ TOSCANO \\ Universidad de Huelva \\ pimia.arteterapia@gmail.com \\ Ma Dolores LÓPEZ MARTÍNEZ \\ Universidad de Murcia \\ fendi@um.es
}

Recibido: 30/03/14

Aceptado: 19/09/14

\section{RESUMEN}

El presente documento ofrece una revisión de programas de arteterapia implementados en las prisiones del mundo, valorando sus efectos dentro del conjunto de los programas de rehabilitación para población reclusa. Ofrece asimismo un informe del programa de arteterapia desarrollado, durante los años 2006 a 2009, en el Centro Penitenciario Huelva II. Se presentan sus objetivos, metodología y resultados de la evaluación cuantitativa y cualitativa de su influencia en los niveles de ansiedad y depresión, así como el efecto obtenido en la reincidencia penitenciaria y en la capacitación sociocultural.

Palabras clave: Arteterapia, evaluación, ansiedad, depresión, reincidencia.

\section{Referencia normalizada}

DOMÍNGUEZ TOSCANO P.Ma, LÓPEZ MARTÍNEZ M ${ }^{\mathrm{a}}$ D. (2014). "Efecto de la arteterapia en la ansiedad y depresión, la capacitación sociocultural y la reducción de la reincidencia penitenciaria de personas reclusas". En Arteterapia: Papeles de arteterapia y educación artística para la inclusión social Vol.: 9. Páginas 39-60. Madrid.

SUMARIO: Introducción. Efecto de los programas de rehabilitación en la reincidencia. Un vistazo a la vida carcelaria. La cara oscura de la moneda. El lado claro de la moneda. Nuevos modelos de intervención. El Currículum de Humanidades. Arteterapia en prisiones. Programa de arteterapia en el Centro Penitenciario Huelva II. Objetivos e Hipótesis de programa de arteterapia. Diseño del programa. Evaluación. Conclusiones. Referencias bibliográficas. 
Effect of art therapy in anxiety and depression, training sociocultural and reducing prison recidivism in inmate

\begin{abstract}
This document provides an overview of art therapy programs implemented in prisons in the world, assessing its effects in rehabilitation programs for prison population. Report also offers art therapy program developed during the years 2006 to 2009, in the prison Huelva II. Objectives, methodology and results of the quantitative and qualitative assessment of their influence on levels of anxiety and depression are presented, and the effect obtained in prison recidivism and the sociocultural training.
\end{abstract}

Key Words: Art therapy, assessment, anxiety, depression, recidivism.

\title{
CONTENTS
}

Introduction. Effect of rehabilitation programs on recidivism. A look at prison life. The dark side of the coin. The light side of the coin. New models of intervention. The Humanities Curriculum. Art therapy in prisons. Art Therapy in the prison Huelva II. Objectives and Hypothesis. Program Design. Evaluation. Conclusions.

\section{Introducción}

En su artículo 25.2, nuestra Constitución establece: "Las penas privativas de libertad y las medidas de seguridad estarán orientadas hacia la reeducación y reinserción social". Esta es la nota común en la mayor parte de los estatutos jurídicos del mundo. En el plano teórico, todos estaríamos de acuerdo en admitir que la privación de libertad debería orientarse con fines reeducativos y de reinserción. Pero, dado que los establecimientos psiquiátricos (manicomios) fueron suprimidos, y dadas las incrementadas carencias asistenciales para inmigrantes y otras poblaciones frágiles, la cárcel termina convirtiéndose en un continente donde van a parar enfermos psiquiátricos, inmigrantes, personas procedentes de entornos desfavorecidos, etc., relegada ya su función teórica de rehabilitar al que delinque.

La cárcel, entendida exclusivamente como castigo, no puede afrontar su nuclear compromiso reeducativo. Una revisión de la literatura sobre reincidencia deja claro que cumplir el final de la condena en sistema de libertad condicional favorece la reinserción de la persona que ha delinquido, mejora un adaptado retorno a la comunidad y reduce la reincidencia. Pero la aplicación de esta medida no sólo no es incompatible sino que se muestra complementaria con otras aplicables intracarcelariamente: los programas de rehabilitación.

\section{Efecto de los programas de rehabilitación en la reincidencia}

No podemos ocultar que existe un extendido pesimismo acerca, no sólo de la pretendida función rehabilitadora de la cárcel, sino incluso de los esfuerzos sistemáticos destinados a la rehabilitación del delincuente. Fundamentados en 
este posicionamiento, encontramos que varios metanálisis que evalúan globalmente los resultados de programas de prevención de la reincidencia o rehabilitación ofrecen conclusiones poco alentadoras: "la evidencia que apoya la eficacia de los programas es poco firme, inconsistente y de fiabilidad dudosa". (Rutter y Giller, 1983, p. 27). Ya desde 1974, los metanálisis realizados por Martinson (1997) sobre los programas de rehabilitación puestos en marcha en las prisiones americanas, dieron como resultado la demostración de la ineficacia de tales programas a efectos de reducir la reincidencia. En los mismos, se mostraron los resultados de los estudios efectuados por Gottfredson (publicados en 1979 y recopilados en 1997), y otros autores que contribuyeron a difundir la impresión de "nada funciona".

Este generalizado pesimismo no debe ocultar que la aplicación de algunos programas específicos sí ha obtenido resultados prometedores. En el contexto argumental de nuestra exposición interesa especialmente explorar el efecto específico de estos programas de rehabilitación, diferenciando este valor del intrínseco a la vivencia de la libertad condicional. Los estudios aportados por Cid y Tébar (2010) coinciden claramente en mostrar que la libertad condicional presenta un efecto reductor sobre la probabilidad de volver a delinquir. Pero lo que aquí interesa particularmente subrayar es que los estudios que analizaban, además, el efecto diferencial de la intervención rehabilitadora concuerdan en destacar la existencia y el valor positivo de este efecto. Centrándonos sólo en aquellas investigaciones que valoran el efecto diferencial de los programas de intervención sobre la reincidencia se observa lo siguiente:

- Se constata la efectividad de los programas de transición entre prisión y libertad (programas fundamentalmente formativos, de educación general y profesional, más los cognitivo-conductuales y de deshabituación) en la reducción de la tasa de reincidencia, según revisión de la literatura sobre este tópico efectuada por Petersilia (2003) en EEUU.

- De la revisión que Seiter y Kandela (2003) emprenden sobre la literatura que describe o evalúa los programas de transición entre prisión y libertad, Cid y Tébar (2010, p. 11) destacan la efectividad de los siguientes programas: "formación profesional y trabajo posterior en el exterior; tratamiento de drogas en prisión, seguido de ayuda y residencia en el exterior; régimen abierto y programas de preparación para la libertad".

- Los trabajos multi-agencia en la comunidad actúan como una adecuada herramienta de seguimiento de delincuentes de alto riesgo, según Worral y Mawby (2004) y Kemshall (2007), en sus descripciones de programas de rehabilitación intensiva para delincuentes de alto riesgo en Inglaterra.

- Los sistemas basados en círculos de voluntarios, orientados a promover un adecuado retorno a la comunidad en delincuentes sexuales con alto riesgo de reincidencia, son efectivos para reducir la reincidencia (Petrunik y Deutschmann, 2008). 
- La intervención mediante programas incrementa la capacidad rehabilitadora de la libertad condicional, haciéndola más efectiva, según Dünkel y Pruin (2010) y Dünkel et al. (2010).

- La supervisión intensiva frente a la supervisión ordinaria no produce diferencias significativas en el efecto contra-reincidencia de la libertad condicional, pero sí lo hace, acentuándolo, la aplicación de programas de intervención (Petersilia y Turner, 1993).

- El efecto reductor de la reincidencia, en delincuentes de alto riesgo liberados condicionalmente, sólo aparece si han sido expuestos a programas de intervención intensos (Solomon et. al, 2005).

- La Libertad condicional y los programas de intervención consiguen disminuir la reincidencia en delincuentes de alto riesgo hasta un $30 \%$ más que en el grupo control equivalente (Braga, Piehl y Hureau, 2009).

Así pues, en la producción científica sobre programas de rehabilitación encontramos una refutación al "nothing works", con respuestas puntuales y específicas a los hándicaps detectados, de modo que podamos cambiar esa expresión fatalista por una opción, no exenta de dificultades, pero impulsada por el convencimiento de que algo se puede hacer.

\section{Un vistazo a la vida carcelaria. La cara oscura de la moneda}

No puede extrañarnos que el encarcelamiento suponga la imposición de un entorno vivencial alienante, cargado de tensiones y aversivo. Consiste en el castigo que nuestro sistema legal (y la mayor parte de los sistemas jurídicos de nuestro entorno cultural) establece para infracciones penales. Intentamos ahora mostrar una breve visión del impacto que el encerramiento ( $\mathrm{y}$ forzada convivencia con personas que también han delinquido) tiene sobre el recluso. Todo ello, con el propósito de perfilar mejor las necesidades de las personas en las que nuestro programa interviene. De este ajuste depende su eficacia.

\section{- Suicidio en prisión}

Las tasas de suicidios en instituciones penitenciarias fluctúan, según estimaciones comparativas con la población no reclusa, entre 5 y 10 veces más que la población de referencia. Tampoco existe acuerdo entre los investigadores sobre si la causa de este hecho radica en la propia situación de encerramiento, con su indudable carga aversiva, o bien la raíz profundiza por debajo del suelo de la prisión para nutrirse de un subsuelo ya predisponente: el presidio se convierte en el habitáculo donde confluyen historias de fracaso múltiple (personal, familiar, social, laboral, etc.). La prisión es sólo la estación terminal (y de frecuente retorno) de un desafortunado trayecto marcado por historiales de desadaptación global. García Marijuán (1997) ofrece una panorámica precisa del suicidio en prisión, tras una revisión de los estudios en ese momento existentes al respecto.

\section{- Depresión}

David Gussak es sin duda el autor más destacado en la promoción y evaluación rigurosa de programas arteterapéuticos en prisiones. De su producción científica hasta la actualidad, la práctica totalidad de documentos se dedica a 
informar sobre programas de arteterapia destinados a reducir la depresión en reclusos. Este autor considera que la depresión y sus consecuencias (fundamentalmente, el suicidio) son el problema de mayor envergadura que afrontan los presos. No es de extrañar que las condiciones de la vida en prisión provoquen la alta prevalencia de esta enfermedad. Las emociones, en este contexto, son manifestaciones de debilidad o vulnerabilidad (que los "reclusos depredadores" aprovechan). La alexitimia o impedimento en la identificación y expresión emocional es considerada un componente fundamental de la depresión, de ahí el éxito de los programas arteterapéuticos en la reducción de esta patología en entornos penitenciarios, pues la creación artística vehicula la expresión de emociones esquivando el engorroso aspecto de "debilidad" asociado a otras formas de manifestar las emociones.

\section{- Ansiedad}

Clemente (1997), tras revisar 90 estudios experimentales sobre los efectos del encarcelamiento encuentra práctica unanimidad en la detección de altos niveles de ansiedad atribuibles a varios factores ansiógenos: el encerramiento, el contacto con "el código carcelario" (el conjunto de normas y costumbres, fuertemente jerarquizadas, establecidas por la sociedad presidiaria), el distanciamiento y frecuente deterioro de lazos afectivos familiares y de pareja, el afrontamiento de peligros que la propia institución penitenciaria no puede controlar (reyertas entre internos, ajustes de cuentas, robos, violaciones). La práctica totalidad de los estudios consultados destacan la ansiedad como la nota común de las personas que viven en reclusión penitenciaria. En varios estudios con población penitenciaria femenina, se informa de la importancia de los sentimientos de culpa en la inhibición de la expresión y la generación de reacciones de ansiedad y depresión. Así lo encontramos en: Fogel y Martin (1992), Anderson et al. (1998), Gallagher, (2000), Ferszt, (2002, 2009).

\section{EI lado claro de la moneda}

Sería injusto, en este vistazo a la vida carcelaria, detenernos exclusivamente en el lado negativo, por más que el sistema penitenciario sea sustancialmente una institución punitiva y toda la terminología al respecto cargue inevitablemente el tinte oscuro de esta realidad.

$\mathrm{Al}$ referirnos a los aspectos positivos de la vida en prisión (que los hay), pensábamos fundamentalmente en las actividades formativas, ocupacionales, recreativas, etc., que el sistema ofrece al interno y que constituyen una buena oportunidad para mejorar la capacitación profesional, incrementar la cultura general, habilidades sociales o recibir tratamiento específico por problemas de adicción, tendencias violentas o sexualidad incontrolada.

\section{Nuevos modelos de intervención: Currículum de Humanidades y Artetera- pia. El Currículum de Humanidades}

La perspectiva humanista adoptada por Jonathan B. King (1986) aporta argumentos para intervenciones que aprovechan el valor de las humanidades en la cons- 
trucción de la identidad histórica, colectiva y cultural del individuo: entiende que la civilización se construye a partir de una trama compleja y múltiple de aportaciones culturales que dota al individuo de riqueza de ópticas y un sentido del ser histórico.

Habitualmente, las personas recluidas en centros penitenciarios presentan estándares educativos bajos, debido fundamentalmente a una escolarización incompleta, absentismo frecuente y bajo rendimiento académico en general. El Currículum de Humanidades como programa de intervención en prisiones fue creado y aplicado por Stephen Duguid y sus colaboradores de la Universidad Simon Fraser, de Canadá, trabajando en conexión con el Servicio Correccional Canadiense. Consiste en la lectura guiada didácticamente y comentada críticamente de textos considerados representativos de la tradición cultural occidental, desde los filósofos griegos hasta literatos actuales, suscitando una vivencia contextual y dramatizada de nuestra herencia intelectual. Garrido (1993, p. 198) comenta en estos términos los resultados del Currículum de Humanidades aplicado en el contexto penitenciario:

Después de casi veinte años de utilización en el estado de Columbia Británica en Canadá, los indicadores de éxito parecen importantes. (...) Así, un estudio cuasi-experimental de este programa encontró una tasa de reincidencia del 16 por 100 para los delincuentes tratados, en comparación con una del 50 por 100 para los internos del grupo de comparación que no recibieron tratamiento (Chaiken, 1989). Por otra parte, un estudio anterior de Ayers, Duguid, Montagye y Wolowidnyk (1980) realizado con alumnos con amplia historia criminal y de adicción a drogas halló resultados parecidos: de los 74 sujetos tratados, sólo reincidió un 14 por 100 en un período de seguimiento de seis meses a cuatro años. Por su parte, la tasa de reincidencia del grupo de comparación fue del 52 por 100.

Las conexiones entre el Currículum de Humanidades y la arteterapia son muchas, pero ésta incorpora una visión multidisciplinar del acopio cultural, e incide en la faceta creadora. De todo ello hablaremos a continuación.

\section{Arteterapia en prisiones}

Una búsqueda reciente ${ }^{1}$, en bases de datos informáticas de documentación bibliográfica, ha obtenido como resultado el hallazgo de 97 documentos sobre aplicaciones arteterapéuticas en prisiones. Los recursos utilizados son: Kvk (Karlsruhe Virtual Catalog), Science Diret, Dialnet, Google Académico, Rebium, Plataforma OVIDSP, Psicodoc, Plataforma Proquest (7 bases de datos), ISI (Web of Knowledge), OCLC (Worlcat), SAGE. Evidentemente, somos conscientes de que estos informes no contemplan la totalidad ni siquiera la mayoría de aportaciones al respecto. No obstante, a tenor de lo encontrado, parece claro que la aplicación

\footnotetext{
${ }^{1}$ Búsqueda realizada en febrero de 2013.
} 
arteterapéutica no es todavía una modalidad de intervención extendida en este tipo de contexto.

En una revisión bibliográfica anterior, ya constatábamos la escasez relativa de investigaciones científicas sobre aplicaciones arteterapéuticas en general. Refiriéndose en concreto a la arteterapia en cárceles, Gussak (2004) declara: "Desafortunadamente, hay poca investigación estructurada y cuantitativa que mida los beneficios de la arteterapia en prisiones". Ésta es una limitación que precisamente ese autor contribuye a reducir. McMillan (2003) también delata la escasa presencia de estudios rigurosamente evaluados en el conjunto de la investigación sobre arteterapia en cárceles. Djurichkoic (2011), en su revisión de la literatura dedicada a evaluar el impacto de los programas arteterapéuticos en las prisiones del mundo, vuelve a pronunciarse en términos parecidos cuando afirma que la historia de la evaluación de programas artísticos en el sector de la justicia penal principalmente consiste en estudios evaluados mediante evidencia anecdótica, relatos personales e informes subjetivos sobre el impacto positivo en el recluso de los proyectos artísticos.

Otra observación que se desprende, tras revisar el conjunto de la literatura científica disponible, es que la arteterapia se muestra susceptible de ser enfocada desde modelos diversos, desde la orientación psicodinámica hasta orientaciones de corte cognitivista, humanista, etc. Pasamos ahora a repasar qué ha ofrecido la arteterapia en el tratamiento de personas reclusas. Por razones de extensión, nos centraremos exclusivamente en los documentos que informan el efecto de la intervención arteterapéutica en la ansiedad y/o depresión del recluso y en la reincidencia tras la excarcelación.

En 1998 aparece, en la revista feminista "Women \& Therapy", un artículo recopilatorio de los resultados de varios programas arteterapéuticos con mujeres reclusas, escrito por B. Merriam. Todos los procesos arteterapéuticos recogidos fueron documentados mediante estudios de caso y trataban mujeres con traumas emocionales severos, depresión y con dificultades para expresarse de manera apropiada.

Gussak encarna una línea de evaluación experimental o cuasiexperimental del efecto arteterapéutico que está siendo paradigmática en este ámbito. En 2003 inicia un estudio en el que se propone medir la efectividad de la arteterapia en el tratamiento de reclusos. Se trata de un estudio cuasiexperimental pre y post-test sin grupo de control, con varones encarcelados. En cuanto a su efectividad, Gussak (2004, p. 246) afirma: "Ha sido demostrado que la acción y la producción artística puede reducir el número de partes disciplinarios imputados a los presos que siguen programas arteterapéuticos (...), y puede ayudar a reducir los síntomas depresivos, una condición que resulta vital en este entorno". El mismo autor informa, dos años más tarde, un estudio de seguimiento al anteriormente informado, también con población reclusa masculina. Corrige la debilidad del estudio cuasiexperimental incorporando un grupo de control, pudiendo comparar los resultados en síntomas depresivos (medidos por el BDI-II) y estrategias expresivas y de socialización (valoradas mediante la escala FEATS) entre el grupo 
experimental, que recibe tratamiento arteterapéutico durante 8 semanas (a sesión por semana) con el grupo control. Utiliza para la valoración comparativa también los informes del equipo de tratamiento de la prisión. En conclusión, corrobora los resultados obtenidos en el estudio piloto de 2004 en relación al descenso en la sintomatología depresiva en el grupo intervenido, decremento significativo en relación al grupo control en el que no se observa reducción alguna. En cambio, la aplicación de la escala FEATS no arroja resultados concluyentes.

Complementa este trabajo con un análisis cualitativo de las transferencias que se producen en el contexto terapéutico entre una interna y su supervisor (Gussak, 2006).

Henk Smeijsters (2006) presenta una revisión del trabajo de 31 arteterapeutas experimentados que trabajan en 12 instituciones penitenciarias holandesas y alemanas. En general, hacen un uso integrado de las artes (plásticas y visuales, danza, música y teatro). Se destaca la importancia de que los internos realicen un trabajo reflexivo y riguroso a partir de la experiencia para obtener resultados claramente favorables en la reducción de la ansiedad y la agresión destructiva.

El trabajo puesto en marcha por Gussak (2007) sobre el efecto de la arteterapia en personas encarceladas con depresión, se traduce en un programa desarrollado en la prisión de North Florida. El programa fue aplicado a 48 reclusos cuyos valores de depresión fueron obtenidos antes y después de la intervención. Su conclusión: "los resultados reflejan un significativo descenso de los síntomas depresivos en aquellos internos que participaron en el programa (de arteterapia)" (Gussak, 2007, p. 444).

Wylie (2007) describe la receptividad de 10 internos al trabajo arteterapéutico grupal. De inspiración claramente psicoanalítica, el trabajo de Wylie vuelve a poner sobre el tapete la ya comentada confusión de límites entre el diagnóstico y la terapia, cuando de expresión artística se refiere.

Vuelve Gussak (2009) a evaluar los efectos de la intervención arteterapéutica, en hombres y mujeres recluidos en instituciones penitenciarias, sobre la depresión, el locus de control y las conductas de socialización. Utiliza como instrumentos de medida The Formal Elements Arts Therapy Scale (FEATS), el Beck Depresión Inventory-Short Form (BDI-II) y el Adult Nowicki-Strickland Locus of Control Scale (ANS), haciendo aplicaciones pre y post al grupo experimental y al grupo control. Los resultados obtenidos respecto a las medidas de depresión y de locus de control son concluyentes; el autor lo expresa en estos términos: "los resultados del BDI-II y del ANS sostienen la asunción de que la arteterapia es efectiva en la reducción de la depresión y en mejorar el locus de control en presos adultos masculinos y femeninos" (Gussak, 2009, p. 5). Como conclusión del conjunto de estudios realizados bajo la coordinación de este investigador, él mismo afirma: "Desde el verano de 2003, varios estudios han sido destinados a cuantificar el beneficio de la arteterapia en la población presidiaria. Estos estudios demuestran una marcada mejora en conducta interpersonal y estrategias de resolución de problemas, así como la efectividad de la arteterapia en la reducción de la depresión en población penitenciaria”. (Gussak, 2009, p. 5). 
Un estudio experimental de Ferszt (2009) arroja resultados positivos acerca del efecto de un programa arteterapéutico con población reclusa femenina, implementado en una prisión del noreste de EEUU. A una muestra de 36 mujeres (21 del grupo de intervención y 15 del grupo control) le fueron medidos los niveles de ansiedad, depresión, autoestima y bienestar espiritual antes y después de la intervención. Los resultados indican que "las participantes en el programa muestran resultados más positivos -que los del grupo comparación- en las entrevistas y en las mediciones cuantitativas de ansiedad, depresión y autoestima" (Ferszt, 2009, p. 47).

La prisión de Grendon viene siendo ampliamente destacada por el uso de técnicas terapéuticas y rehabilitadoras de amplio espectro. Desde hace más de 30 años se viene utilizando la dramaterapia y el psicodrama como recursos terapéuticos que han demostrado su eficacia en la mejora de los reclusos, particularmente en cuanto a habilidades sociales. J. Jefferies (2010) ofrece un informe sobre el arraigo neurológico del trabajo con roles y sus aplicaciones en contexto penitenciario. También contextualizado en Grendon, B. Wyllie (2010) informa un programa arteterapéutico evaluado con una metodología cualitativa, partiendo del análisis detallado de las imágenes. La inspiración psicoanalítica de los análisis así como de la concepción de la interpretación es evidente.

Un estudio de obligada referencia es el metanálisis ofrecido por B. Meekums y J. Daniel (2010), en el que hace una recopilación exhaustiva, revisión y valoración crítica de la producción científica sobre arteterapia aplicada a delincuentes. Revisa documentos que informan investigaciones realizadas con metodología tanto cuantitativa como cualitativa. En palabras de los autores, "las terapias artísticas han sido sistemáticamente asociadas a la mejora en los niveles de arousal $^{2}$, equilibrio emocional y calidad de vida... Los autores recomiendan una aproximación que utilice metodología mixta, a fin de facilitar tanto la detección como la comprensión de los efectos de las artes en delincuentes... y la conducta agresiva" (Meekums y Daniel, 2010, p. 229).

Centrado en dramaterapia, sale a la luz en 2011 un interesante libro: Performing new lives: Prison theatre, recopilatorio de experiencias teatrales en las prisiones del mundo anglosajón. Entre los múltiples programas informados se encuentran, por ejemplo, el "Shakespeare Behind Bars" desarrollado en LaGrange, prisión de Kentucky, o el "ArtsAloud" implementado en Austin, Texas. Los autores del texto aportan diversas visiones sobre la contribución de la terapia teatral al fortalecimiento de recursos para la mejora personal y social, y el equilibrio emocional (reducción de ansiedad y depresión) en las cárceles.

La exposición de Djurichkoic (2011) ofrece una panorámica de los programas arteterapéuticos en prisiones fundamentalmente de Australia y EEUU. Destaca que, a pesar de la relación de investigaciones que informan de éxitos terapéuticos

2 “Arousal" se suele traducir por "activación". 
a través del arte en contexto penitenciario, no existe en la mayoría de países una política estatal que incluya formalmente y estructure las prácticas arteterapéuticas en estos entornos.

Para una visión precisa de la importancia del trabajo interdisciplinar entre psicólogos y arteterapeutas en la rehabilitación del delincuente, es de lectura obligada el artículo de Breiner, M., Tuomisto, L., Gussak, D. y Aufderheide, D. (2012). En él se describen las prácticas de los graduados en arteterapia por la Universidad de Florida, trabajando en prisiones de ese Estado. El interés principal de este artículo, a nuestro entender, radica en la visión que ofrece sobre cómo integrar la terapia cognitivo-conductual y la arteterapia en prosecución del objetivo común de controlar la ira y las conductas antisociales en reclusos.

Para consultar un informe detallado sobre el trabajo arteterapéutico, sesión por sesión, con delincuentes afectados por patología psiquiátrica, se destaca el capítulo de Ph. Cronin (2012) integrado en un libro sobre evaluación en arteterapia.

\section{Programa de Arteterapia en el Centro Penitenciario Huelva II}

Tras el recorrido realizado, se presenta, a continuación, una investigación arteterapéutica dentro del contexto penitenciario español. Se trata de la aplicación de un programa de arteterapia desarrollado con un grupo de treinta y dos reclusos durante 3 años, desde 2006 a 2009. Siguiendo el modelo de Villabona (Asturias), en el Centro Penitenciario Huelva II se implantó, en el Módulo 6, una UTE (Unidad Terapéutica Educativa) articulada en torno al objetivo fundamental de la prisión como espacio rehabilitador. La UTE sirvió de receptáculo para desarrollar este programa de arteterapia.

\section{Objetivos e hipótesis del programa de Arteterapia}

- Reducir la reincidencia en personas reclusas, una vez sean excarceladas.

- Reducir la ansiedad y la depresión.

- Habilitar recursos del desarrollo cultural como función mediática en el intercambio social, favoreciendo una valoración positiva de la propia aportación como fuente de enriquecimiento colectivo.

Entre las hipótesis para variables cuantificables podemos formular la hipótesis $\mathrm{n}^{\circ} 1$ : Bajo la hipótesis alternativa, se espera obtener diferencias estadísticamente significativas entre la tasa de reincidencia de los reclusos participantes en el programa, una vez excarcelados, y la tasa española de reincidencia.

Hipótesis $\mathrm{n}^{\circ}$ 2: Se espera obtener diferencias estadísticamente significativas en los resultados medidos del nivel de Ansiedad Estado y Ansiedad Rasgo tras la intervención en el grupo experimental y la no intervención en el grupo control, con un nivel de significación de $\alpha=0.05$.

Hipótesis $\mathrm{n}^{\mathrm{o}}$ 3: Se espera obtener diferencias estadísticamente significativas en los resultados medidos del nivel de Depresión tras la intervención en el grupo experimental y la no intervención en el grupo control, con un nivel de significación de $\alpha=0.05$. 


\section{Diseño del programa}

- Enfoque Metodológico del proceso empírico

Tras el rastreo documental se pasó a la realización de un trabajo de campo de carácter exploratorio y de enfoque fundamentalmente cuantitativo. Para la recogida de datos se utilizaron los siguientes instrumentos:

Para la medición de la ansiedad se aplicó el STAI, adaptación española realizada por Nicolás Seisdedos, editado por TEA Ediciones. Para la medida de la depresión se empleó la adaptación española a la segunda versión del Beck Depression Inventory (BDI-II), hecha por Sanz y Vázquez (2011). Este instrumento ha sido diseñado para evaluar la presencia y gravedad de los síntomas depresivos consistentes con el DSM-IV.

- Muestra:

Los sujetos participantes son hombres recluidos en el Centro Penitenciario Huelva II, módulo 6, en régimen penitenciario de primer o segundo grado. El tipo de delito por el que ingresaron en prisión es variado. La duración de las condenas de los participantes que completaron el estudio también presenta un amplio rango, desde 5 años hasta 40 años. El rango de edades, al comienzo del estudio, oscila entre 18 y 52 años. El grupo se formó con un total de treinta y dos participantes divididos en un grupo control compuesto por 16 sujetos y un grupo experimental con 16 sujetos. Dado que la participación es necesariamente voluntaria, y que la formación de ambos grupos corrió a cargo del Equipo de Tratamiento, no se puede afirmar a priori que ambos estén equiparados en las variables dependientes medibles en ese momento (ansiedad, depresión) y en variables potencialmente extrañas que pudieran incidir en las anteriores (factores de personalidad, tratamientos farmacológicos, etc.). Para comprobar que ambos grupos son comparables e inicialmente semejantes en las variables de interés (así como en la incidencia sobre éstas de posibles variables perturbadoras), se recurre a métodos estadísticos, aplicables a posteriori de la formación de grupos; utilizamos concretamente el contraste $t$ de Student para grupos independientes.

- Descripción del programa ${ }^{3}$

Desde el comienzo se procuró que los participantes tuviesen claro que no estaban en un taller para el aprendizaje de técnicas artísticas, y que su actitud debía enfocarse a la recalificación ética de los actos cometidos, a un fortalecimiento de su conciencia relacionada con la capacidad para el cambio, a la intención de ir definiendo los términos de sus propias opciones vitales y el deseo de emprender una vida ajena al delito y sus consecuencias. De este modo, las posiciones quedaron bien perfiladas desde el inicio con el fin de evitar las menores bajas posibles.

\footnotetext{
${ }^{3}$ Para una descripción más completa y detallada del contenido del programa se puede consultar la publicación: DOMÍNGUEZ-TOSCANO, P.M. (2014). El arte como constructor de paz social. Huelva: Svo. de Publicaciones de la Universidad de Huelva.
} 
El programa se compuso por once bloques temáticos relacionados con el ingreso en prisión y la vida durante y después de la condena. Todos los bloques incluyen un total de cincuenta actividades donde se intentaron trazar las guías de orientación y el cauce hacia la progresiva afloración de asuntos que fuesen marcando el sentido del tratamiento. A continuación se muestra un resumen del mismo:

- Bloques 1 y 2: Contenidos: Prolegómenos: presentación del programa y los participantes; construyendo el grupo. Proyecciones. Técnicas: Se realizaron técnicas de relajación (respiratorias, musculares y de danzaterapia), técnica de garabateo-desgarabateo y de proyección literaria en la que se trataban diversas cuestiones relacionadas con la vida en prisión. Todas las técnicas eran seguidas de debate sobre la experiencia y los contenidos de la misma.

- Bloque 3: Contenidos: Utilización de recursos plásticos, simbólicos y de la cultura visual. Técnicas: se aplicaron técnicas de estampación inversa, de muy fácil ejecución, con pintura sintética sobre agua, imprimaciones de témpera sobre papel, collage con y sin textura, interpretación visual de emociones y sensaciones no visuales (pintar emociones a partir de una autoinducción emocional con y sin música, interpretaron visualmente sensaciones táctiles). Los talleres "de la mano de..." proporcionan una toma de contacto con la vida y obra de conocidos creadores plásticos $\mathbf{y}$ escritores que, experimentando situaciones difíciles, hicieron del conflicto arte o literatura. Este acercamiento a la creación mediante la provocación de nexos empáticos con los creadores, es seguida de una presentación de algunos elementos constitutivos de la cultura visual actual, procedentes del arte contemporáneo y la publicidad.
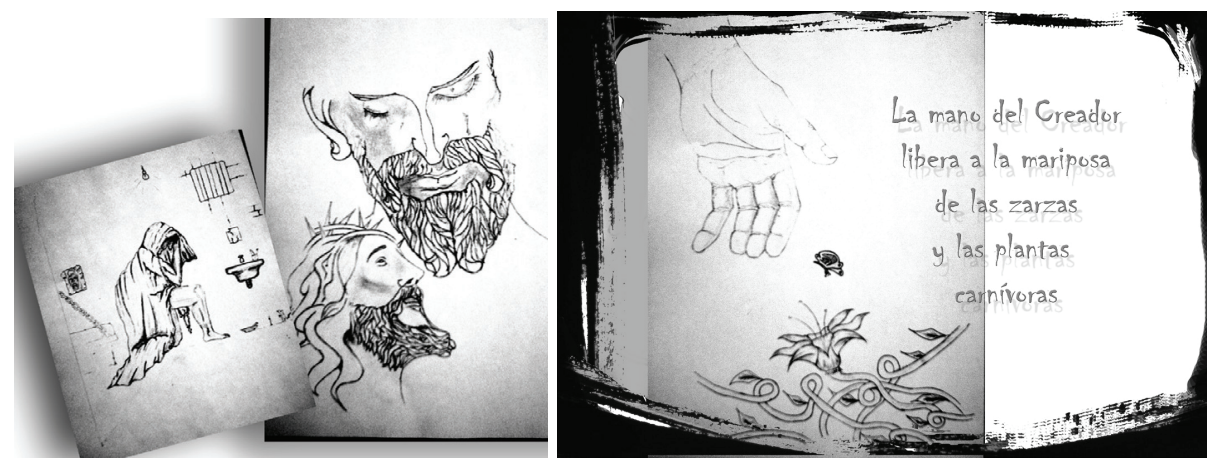

Fig.1. y Fig. 2. Con libertad de técnica y tema J.A. Delgado expresó y condensó simbólicamente su experiencia del arrepentimiento y deseos de reconstrucción personal.

- Bloques 4 y 5: Contenidos: Construcción de autoimagen positiva, reforzamiento de la percepción de autoeficacia y gestión del impacto emocional que produce la reclusión. Identificación, valoración y afrontamiento arteterapéutico del efecto que producen los factores ansiógenos o depresivos relacionados con la propia 
situación de reclusión: deterioro o ruptura de pareja, incumplimiento forzoso o limitación en el cumplimiento de obligaciones familiares (sostenimiento económico, apoyo a la atención educativa, fortalecimiento de lazos afectivos, generación de confianza, etc.), ingreso en la subcultura carcelaria, con estructura jerárquica, códigos éticos y conductuales propios; convivencia con otras formas de delincuencia; limitación de recursos para gestionar productivamente el tiempo. Técnicas: Escenificaciones teatrales, debate grupal sobre asuntos relacionados con la realidad penitenciaria, lectura de escritores presos, creación plástica y literaria.

- Bloques 6 y 7. Contenidos: A) Recalificación ética del delito como error: Este apartado se dividió en cuatro fases: Fase 1: Análisis de los factores coadyuvantes en el delito. Fase 2: Empatización con la o las víctimas. Fase 3. Gestión de la autoinculpación y arrepentimiento. Fase 4: Consideración de alternativas al delito. B) Adaptación al sistema penitenciario: revisar las actitudes ante la sentencia. Técnicas: representaciones teatrales recreando actos delictivos, tras analizar los elementos presentes antes de la comisión del acto (factores predisponentes y desencadenantes) y las consecuencias del mismo; expresión escrita; para la empatización con las víctimas se utilizó la visualización de imágenes creadas por víctimas de cualquier forma de violencia, lectura de textos donde víctimas relatan su experiencia. Estas actividades, altamente tensionantes, son seguidas de expresión plástica de emociones, pensamientos y reflexiones suscitados. Se empieza a trabajar la consideración de alternativas al delito y una autopercepción futura libre de delincuencia. Técnicas: dramatizaciones "Dando voz a posibles víctimas futuras", "Si aquel día yo no...", estructuración del pensamiento en torno al delito, con expresa consideración de alternativas al mismo mediante mapas conceptuales, "relatos en la comba", reflexión colectiva y debates guiados en torno a la capacidad de elegir y la responsabilidad de elegir, más una evaluación ajustada de los costes. Finalmente, preparando una mejor integración del recluso en la vida penitenciaria, y que éste consiga maximizar el aprovechamiento de los recursos educativos, culturales, etc. que la prisión proporciona, se trabaja plástica y literariamente la propia evaluación que los reclusos hacen de la administración de justicia, "mi sentencia de la sentencia" a fin de que esta valoración consciente y expresada favorezca tomas de conciencia más realistas y objetivas, con atribución más internalizadas de responsabilidades (evitando la habitual externalización de causas), y una actitud constructiva.

- Bloque 8. Contenidos: Fortalecer la imaginación. Construyendo espacios de nueva realidad. Técnicas: "¿Tú qué ves? (¿Tú qué miras?)”, "Cadáver exquisito”, "De la mano de los surrealistas", "De la mano de mi propia imaginación".

-Bloque 9. Contenidos: La vida después de la prisión. Técnicas: Debate guiado sobre la vida tras el cumplimiento de condena, habilidades para afrontar los juicios sociales paralelos y el retorno a entornos criminógenos. Reflexión com- 
partida sobre la confrontación de valores mantenidos en entornos criminógenos y la sociedad en su conjunto. Se aborda la problemática de la inserción familiar, social y laboral, la necesidad de romper el estigma y favorecer el acceso al mundo sociolaboral mediante estas técnicas: dramatizaciones para el fortalecimiento de habilidades sociales; aprendizaje de habilidades sociales que ayuden a mejorar la vida familiar; simulaciones escenificadas de situaciones: A) Relacionadas con el acceso al mundo laboral, B) Situaciones sensibles en las relaciones interpersonales, C) Simulación de situaciones sensibles en las relaciones familiares. La mirada preactiva.

- Bloque 10 y 11. Contenidos: Renovarse, con arte. Exponernos. Técnicas: "Sentir el color del aire detrás de los muros". "La renovación de las águilas" (creación literaria". "Renacimiento" (creación pictórica). Murales colectivos sobre paneles de madera de grandes dimensiones. Exponernos públicamente: Recital de poesía compuesta por los participantes; exposición de obra plástica; intercambio en la red; publicación de libros de autoría colectiva ${ }^{4}$.

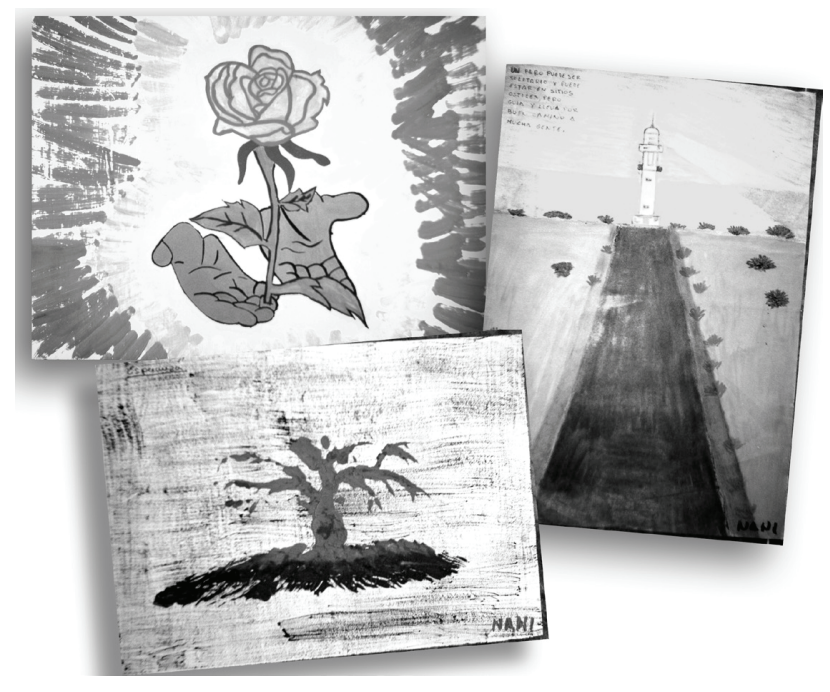

Fig. 3. Obras de Nani, producidas en el bloque 10: Renovarte con arte. Sus comentarios: "Para María", "Árbol aparentemente seco, pero está en tierra fértil y pronto rebrotará", "Un faro puede ser solitario y puede estar en sitio hostiles, pero lleva luz por el buen camino a mucha gente".

4 Ejemplos: El libro: "La libertad construida" recoge una selección de la producción literaria surgida en el taller de arteterapia desde 2006 a 2008. En 2010 sale a la luz otro libro, "Cartas en el patio", de autoría compartida entre 5 reclusos. Ambas publicaciones suponen para los autores un importante estímulo, fortalece su conciencia de formar parte de una sociedad que valora su esfuerzo y su obra, y los asume como agentes activos del propio tejido cultural. 


\section{Evaluación}

- Evaluación del objetivo 1 (reducir la reincidencia)

El objetivo fundamental de este programa es la reducción de la reincidencia, cuya tasa española se establece como promedio en el $55 \%$; hablamos de reincidencia penitenciaria (comisión de nuevo delito, detención, enjuiciamiento y condena a privación de libertad). Entre los reclusos atendidos con arteterapia, durante al menos un año ininterrumpido de los tres de duración total del programa, la tasa se redujo al $12,5 \%$ en los cuatro años siguientes tras la terminación del programa y, en función de sus fechas de puesta en libertad, al año, a los dos o a los tres años tras el cumplimiento de condena (según los casos).

Para comprobar si la diferencia respecto a la tasa nacional es significativa, aplicamos $X^{2}$ de Pearson. Obtenemos una $X^{2}=5,25$. Para un nivel de significación del .05 y 1 grado de libertad (g.l.), nuestro valor supera el valor crítico. Por tanto, podemos afirmar que las diferencias entre los resultados obtenidos por nuestra muestra, en cuanto a nivel de reincidencia, y lo esperado a partir de la tasa nacional de reincidencia, son significativamente diferentes.

- Evaluación del objetivo 2 (reducir la ansiedad y la depresión)

En relación con los resultados relacionados con la ansiedad (medida mediante el STAI) de los participantes se muestran los siguientes resultados:

\begin{tabular}{|c|c|c|c|c|c|c|c|c|}
\hline & \multicolumn{4}{|c|}{ Control } & \multicolumn{4}{|c|}{ Experimental } \\
\hline & \multicolumn{2}{|l|}{ Pre } & \multicolumn{2}{|l|}{ Post } & \multicolumn{2}{|l|}{ Pre } & \multicolumn{2}{|l|}{ Post } \\
\hline & Estad & Rasgo & Estad & Rasgo & Estad & Rasgo & Estad & Rasgo \\
\hline Media & 26.03 & 25.01 & 25.78 & 25.12 & 26.11 & 24.78 & 21.09 & 22.75 \\
\hline $\begin{array}{l}\text { Desviación } \\
\text { Típica }\end{array}$ & 1.69 & 1.60 & 1.81 & 1.73 & 1.75 & 1.83 & 1.62 & 1.74 \\
\hline Edad & $19-52$ & $19-52$ & $20-53$ & $20-53$ & $18-49$ & $18-49$ & $19-50$ & $19-50$ \\
\hline
\end{tabular}

Tabla $^{\circ} 1$. Resultados sobre la ansiedad de los participantes.

Dado que las muestras no han podido formarse por aleatorización, y que los criterios utilizados por el equipo tratamental del Centro Penitenciario para la formación de los grupos pudieran incidir en los resultados del programa, utilizamos la ecuación $t$ de Student para dos muestras independientes para valorar si las diferencias encontradas en las medidas pre son estadísticamente significativas.

T1E (contraste medidas pre en Ansiedad Estado a grupos control y experimental) $=-0.22$. Para 30 g.l. y nivel de significación .05 , nuestro valor está por debajo del valor crítico $t$ (2.04) para pruebas de dos direcciones. Por tanto, se puede afirmar que ambas muestras (experimental y control) proceden de la misma población.

T1R (contraste medidas pre en Ansiedad Rasgo a grupos control y experimental $)=0.36$. Para 30 g.l. y nivel de significación .05 , nuestro valor está por debajo del valor crítico $t$. De este modo, aun sin aleatorizar la extracción de las muestras, podemos utilizarlas como equivalentes en cuanto a la medida pre de Ansiedad Rasgo y de Ansiedad Rasgo, resultando razonablemente válido compararlas. 
T2E (contraste medidas post en Ansiedad Estado a grupos control y experimen$\mathrm{tal})=7.45$; T2R (contraste medidas post en Ansiedad Rasgo a grupos control y experimental $)=3.73$. Para 30 g.l. y nivel de significación .05 el valor crítico $t$ para pruebas de dos direcciones es 2.04. Dado que ambos valores obtenidos (T2E y T2R) superan el valor crítico, podemos afirmar que los resultados obtenidos por los grupos experimental y control en Ansiedad Estado y en Ansiedad Rasgo son significativamente diferentes.

- Depresión

Tanto los sujetos experimentales como los del grupo control cumplimentaron el BDI-II antes y después de la intervención.

\begin{tabular}{|l|l|l|l|l|}
\hline & \multicolumn{2}{|l|}{ Control } & Experimental \\
\cline { 2 - 5 } & Pre & Post & Pre & Post \\
\hline Media & 18.46 & 19.12 & 19.06 & 17.15 \\
\hline $\begin{array}{l}\text { Desviación } \\
\text { Típica }\end{array}$ & 1.98 & 1.42 & 1.87 & 1.65 \\
\hline Edad & $19-52$ & $20-53$ & $18-49$ & $19-50$ \\
\hline
\end{tabular}

Tabla n². Resultados sobre la depresión de los participantes.

T1 (contraste medidas pre en Depresión a grupos control y experimental $)=$ 1.45. Para 30 g.l. y nivel de significación .05, el valor crítico $t$ (2.04) está por encima del obtenido en nuestras muestras. Concluimos que pueden ser utilizadas como equivalentes en cuanto a la medida pre de Depresión, resultando razonablemente válido compararlas.

T2 (contraste medidas post en Depresión a grupos control y experimental $)=$ 3.62. Para 30 g.l. y nivel de significación .05 el valor crítico $t$ está por debajo del obtenido en nuestro estudio. Podemos, por tanto, afirmar que los resultados obtenidos por ambos grupos son significativamente diferentes.

En conjunto, se puede afirmar que el programa de arteterapia ha reducido significativamente la Ansiedad Estado, Ansiedad Rasgo y Depresión en los reclusos participantes en el mismo.

- Evaluación del objetivo $3^{\circ}$

El programa se marcó otras dianas de la intervención, evaluadas cualitativamente.

Como exponente de la generación o fortalecimiento de habilidades de expresión artística, comunicativa y de habilitación cultural (objetivo 3), podemos mencionar la realización y publicación de dos libros de los que son autores reclusos participantes en el proyecto: "La libertad construida" y "Cartas en el patio", ya citados. La evaluación del cumplimiento de este objetivo se hace mediante autoinforme de los participantes e información obtenida de la psicóloga del módulo.

\section{Conclusiones}

Tras la revisión documental realizada sobre investigaciones de arteterapia aplicadas en centros penitenciarios queda constancia de la laguna existente en cuanto a 
producción científica. De acuerdo con Gussak (2004) y Djurichkoic (2011), actualmente hay poca investigación estructurada que mida el efecto de la arteterapia en las prisiones y lo que se encuentra es un predominio de estudios sobre programas evaluados mediante evidencias anecdóticas, relatos personales e informes subjetivos sobre el impacto en el recluso de los proyectos artísticos. No obstante, existe literatura científica al respecto donde se informan resultados positivos obtenidos por las terapias artísticas en los niveles de ansiedad y, sobre todo, de depresión del recluso.

Los resultados obtenidos con el programa de arteterapia implementado en el Módulo VI del Centro Penitenciario Huelva II, durante los años 2006 al 2009, demuestran que se ha conseguido reducir significativamente el nivel de depresión y ambos tipos de ansiedad en los sujetos participantes en el programa. Esta reducción es mayor en la Ansiedad Estado que en la Ansiedad Rasgo. Se ha producido también, y de manera destacable, una reducción de la reincidencia en los participantes que han sido excarcelados durante el periodo de seguimiento de 2009 a 2013.

La escasez de programas implantados y la aplicación de este programa de arteterapia invitan a una reflexión crítica de cara a futuras implementaciones. Es necesario articular tácticas terapéuticas específicas para reducir los partes disciplinarios o el tipo de situaciones incidentales que conducen a la aplicación de traslados intermodulares; ello nos ha llevado a un análisis más detenido de las causas habituales de tales traslados, así como una elaboración de actividades específicamente tendentes a propiciar una toma de conciencia por parte de los reclusos sobre la importancia de mejorar la vida carcelaria en este aspecto. Es preciso asimismo procurar de las autoridades penitenciarias una mayor implicación con el programa, de modo que sea valorada la auténtica necesidad de aplicar traslados intermodulares a los reclusos participantes, especialmente en determinadas fases del proceso terapéutico. Hecha una valoración conjunta, y teniendo como principales elementos de juicio la reducción de la reincidencia, de la ansiedad y la depresión y la participación de los excarcelados en la vida cultural de sus respectivas comunidades, los resultados obtenidos animan a futuras implementaciones de programas de arteterapia en las prisiones españolas.

Para finalizar aprovechar este espacio para hacer nuevamente público nuestro agradecimiento al Equipo de Tratamiento, a la Dirección del Centro pero, muy especialmente, a los educadores del Módulo 6, José Enrique Allepúz y Ma Luisa Hernández. Su apoyo decidido al programa de AT ha permitido limar los obstáculos -nada despreciables- de la burocracia penitenciaria. Sin ellos, sin su entusiasmo y su convicción de que la cárcel puede convertirse en oportunidad para el cambio, esta experiencia sería sencillamente inimaginable. 


\section{Referencias bibliográficas}

BENNINK, J., GUSSAK, D. E., \& SKOWRAN, M. (2003). "The role of the art therapist in a Juvenile Justice Setting". En: The Arts in Psychotherapy, 30(3), 163-173.

BREINER, M., TUOMISTO, L., BOUYEA, E., GUSSAK, D. Y AUFDERHEIDE, D. (2012). "Creating an Art Therapy Anger Management Protocol for Male Inmates Through a Collaborative Relationship". En: International Journal of Offender Therapy and Comparative Criminology, 56, 1-20.

BREWSTER, L.G. (1983). An evaluation of the Arts-in-Corrections Programs of de California Department of Corrections. San Jose, CA: San Jose State University.

CRONIN, PH. (2012). "Art therapy assessment of adults in an inpatient forensic setting”. En: Assessment in art therapy. New Yord: Routledge/Taylor \& Francis Group, 116-119.

DJURICHKOIC. (2011). Arts in Prisons. A literature review of the philosophies and impacts of visual arts programs for correctional populations. Australia: UTS Shopfront Student Series.

DOMÍNGUEZ-TOSCANO, P.M. (2014). El arte como constructor de paz social. Huelva: Svo. de Publicaciones de la Universidad de Huelva.

GARRIDO GENOVÉS, V. (1993). Técnicas de tratamiento para delincuentes. Madrid: Editorial Centro de Estudios Ramón Areces, S.A.

GUSSAK, D. \& VIRSHUP, E. (Eds.) (1997). Drawing Time: Arte Therapy in Prisons And Other Correctional Settings. Chicago, I.L.: Magnolia Street Publishers.

GUSSAK, D. (2007). "The Effectiveness of Art Therapy in Reducing Depression in Prison Populations". En: International Journal of Offender Therapy and Comparative Criminology, 51 (4), 444-460.

MEEKUMS Y J. DANIEL (2010). "Arts with offenders: A literature synthesis". En: The Arts in Psychotherapy, 38 (4), 229-238.

SMEIJSTERS, H., VAN DEN BRAAK, J., HELMICH, M., REUMERS, H., \& VAN DER WEKKEN, J. (2009). (Arts therapies in judicial institutions and locked care for youth) Vaktherapie in de Justitiele Jeugdinrichtingen en de Gesloten Jeugdzorg. Kaderdocument voor alle vaktherapieën. Heerlen/Cadier $\&$ Keer. The Netherlands: KenVaK/Het Keerpunt. 


\section{Bibliografía ampliada (sólo sobre arteterapia en prisiones)}

ARGUE,J.; BENNETT,J. Y GUSSAK, D. (2009).Transformation through negotiation: Initiating the Inmate Mural Arts Program. The Arts in Psychotherapy, 36, 313-319.

AYLLÓN, T. Y MILAN, M.A. (1979). Correccional rehabilitation and management. N.Y.: Wiley.

BAYÓN, F; COMPADRE, A. Y SALARICH, L (1997). Programas de desarrollo de la competencia social. En M. Clemente y J. Núñez: Psicología Jurídica Penitenciaria II, 37-74.

BREINER, M., TUOMISTO, L., BOUYEA, E., GUSSAK, D. Y AUFDERHEIDE, D. (2012). Creating an Art Therapy Anger Management Protocol for Male Inmates Through a Collaborative Relationship. International Journal of Offender Therapy and Comparative Criminology, 56, 1-20.

BREWSTER, L.G. (1983). An evaluation of the Arts-in-Corrections Programs of de California Department of Corrections. San Jose, CA: San Jose State University.

CAPDEVILA CAPDEVILA, M. Y FERRER PUIG, MARTA (2009). Tasa de reincidencia penitenciaria 2008. Barcelona: Centro de Estudios Jurídicos y Formación Especializada.

CLEMENTE, M. (1997). Los efectos psicológicos y psicosociales del encarcelamiento. En M. Clemente y J. Núñez: Psicología Jurídica Penitenciaria II, 383-408.

CRONIN, PH. (2012). Art therapy assessment of adults in an inpatient forensic setting. En Assessment in art therapy. New Yord: Routledge/Taylor \& Francis Group,116-119.

DJURICHKOIC (2011). Arts in Prisons. A literature review of the philosophies and impacts of visual arts programs for correctional populations. Australia: UTS Shopfront Student Series.

DOMÍNGUEZ-TOSCANO, P.M (Coord.) (2009b). La libertad construida. Sevilla: Ituci Siglo XXI.

(Coord.) (2010). Cartas en el patio. Sevilla: A. C. Ediciones Océano.

(2014). El arte como constructor de paz social. Huelva: Svo.Publicaciones de la Universidad de Huelva.

DUITS, N., VAN CASTEREN, M., VAN DEN BRINK, W., \& DORELIJERS, TH. A. H. (2005). Risicotaxatie van geweldsrecidive bij jeugdigen [Risk taxation of violence recidivism by youngsters]. Tijdschrift voor psychiatrie, 47, 511-518.

FARRINGTON, D. ET AL. (1988). Are there any successful men from criminognic backgrounds? Psychiatry, 51, 116-130. 
FENNER, L.B.; GUSSAK, D. (2006). Therapeutic Boundaries in a Prison Setting: A Dialogue between an Intern and her Supervisor. The Arts in Psychoterapy, 33, 414-421.

FERSZT, G.; SALGADO, D; DEFEDELE, S. Y LEVEILlEE, A.. (2009). Houses of Healing: A Group Intervention for Grieving Women in Prison. The Prison Journal 89 (1), 46-64.

GARCÍA MARIJUÁN, J.A. (1997). Programas de prevención de suicidios en prisión. En Clemente, M. y Núñez, J.: Psicología Jurídica Penitenciaria II, 199-217.

GARDSTROM, S. C. (1999). Music exposure and criminal behavior: Perceptions of juvenile offenders. Journal of Music Therapy, 36(3), 207-221.

GARRIDO GENOVÉS, V. (1993). Técnicas de tratamiento para delincuentes. Madrid: Editorial Centro de Estudios Ramón Areces, S.A.

GIBBS, J., POTTER, G., \& GOLDSTEIN, A. (1995). The Equip Program. Teaching youth to think and act responsibly through a peer-helping approach. Champaign, IL: Research Press.

GUSSAK, D. (1997). Breaking through barriers: Art Therapy in Prisons. En D. Gussak \& E. Virshup (Eds.): Drawing Time: Arte Therapy in Prisons And Other Correctional Settings. Chicago, I.L.: Magnolia Street Publishers, 1-11.

GUSSAK, D. (2004). Art Therapy with Prison Inmates: a Pilot Study. The Arts in Psychoterapy, 31, 245-259.

GUSSAK, D. (2006a). Effects of Art Therapy with Prison Inmates: A Follow-up Study. The Arts in Psychoterapy, 33, 188-198.

GUSSAK, D. (2006b). Therapeutic boundaries in a prison setting: a dialogue between an intern and her supervisor. The Arts in Psychotherapy, 33, 414-421.

GUSSAK, D. (2007). The Effectiveness of Art Therapy in Reducing Depression in Prison Populations. International Journal of Offender Therapy and Comparative Criminology, 51 (4), 444-460.

GUSSAK, D. (2009a). The effects of art therapy on male and female inmates: Advancing the research base. The Arts in Psychotherapy, 36, 5-12.

GUSSAK, D. (2009b). Comparing the effectiveness of art therapy on depression and locus of control of male and female inmates. The Arts in Psychotherapy, doi. 10.1016/j.aip.2009.02.004

HENK SMEIJSTERS, PHD. ET AL. (2011). Arts therapies for young offenders in secure care: A practice-based research. The Arts in Psychotherapy 38, 4151.

JEFFERIES, J. (2010). Psychodrama as part of core therapy at HMP Grendon. En Grendon and the emergence of forensic therapeutic communities: Developments in research and practice. Londres: Wiley-Blackwell. 
KIPPER, D. A., \& RITCHIE, T. D. (2003). The effectiveness of psychodramatic techniques: A meta-analysis. Group Dynamics: Theory, Research and Practice, 7, 13-25.

KORNFELD, P. (1997). Cellblock vision: Prison Art in America. Princeton, NJ: Princeton University Press.

KURSTJENS, H., \& BAERENDS, A. (2006). Actieve muziektherapie. In H. Smeijsters (Ed.), Handboek muziektherapie. Evidence-based practice voor de behandeling van psychische stoornissen, problemen en beperkingen (Handbook of music therapy. Evidence-based practice for the treatment of psychic disturbances, problems and limitations). Houten, The Netherlands: Bohn Stafleu Van Loghum.

LEVY, B. (1978). Art Therapy in a Women's Correctional Facility. Art Psychotherapy, 5, 157-166.

LITTEL, G. Y ROBINSON, K. (1986a). Discovering life and liberty in the pursuit of happiness. Memphis, TN: Eagle Wing Books.

_ (1986b): How to escape your prison. Memphis, TN: Eagle Wing Books.

LODEWIJKS, H. P. B., DORELEIJERS, TH. A. H., DE RUITER, C. DE, \& DE WIT-GROULS, H. (2003). (Structured taxation of violence recidivism by youngsters) Gestructureerde taxatie van geweldsrisico bij jongeren. Eefde. The Netherlands: Rentray.

MARCUS-MENDOZA, S. Y WRIGHT, E. (2004). Descontexualizing Female Criminality: Treating Abused Women in Prison in the United States. Feminism Psychology, 14 (2), 250-255.

MCMURRAY, M., \& SCHWARTZ-MIRMAN, O. (1998). Transference in art therapy: A new outlook. The Arts in Psychotherapy, 25, 31-36.

MEEKUMS Y J. DANIEL (2010). Arts with offenders: A literature synthesis. The Arts in Psychotherapy, 38 (4), 229-238.

MERRIAM, B. (1998). To Find a Voice: Art Therapy in a Women's Prison. Women \& Therapy, 21(1), 157.

MILLIKEN, R. (2002). Dance/movement therapy as a creative arts therapy approach in prison to the treatment of violence. The Arts in Psychoterapy, 29, 203-206.

PERSONS, R. W. (2009). Art therapy with serious juvenile offenders: A phenomenological analysis. International Journal of Offender Therapy and Comparative Criminology, 53, 433-453.

PLATT, J.J. Y PROUT, M. (1987). Cognitive-Behavioral theory and interventions for crime and delinquency. En E.K. Morris y C.J. Brauckmann (Eds.): Behavioral approaches to crime and delinquency. N.Y.: Plenum, 477-497.

SMEIJSTERS, H. (Ed.). (2009b). Research in practice in the arts therapies. Heerlen, The Netherlands: KenVaK/Melos. 
URSPRUNG, W. (1997). Insider art: The creative ingenuity of the incarcerated artist. En D. Gussak \& E. Virshup (Eds.). Drawing Time: Art Therapy in Prisons And Other Correctional Settings (pp. 13-24). Chicago, I.L.: Magnolia Street Publishers.

VVAA (2011). Performing new lives: Prison theatre. Londres: Jessica Kingsley Publisher.

WYLIE, B. (2007). Self and social function: Art Therapy in a therapeutic community prison. Journal of Brand Management, 14 (4), 324-355. 